\title{
Biogeógrafos del mundo... ¡uníos!: un camino hacia la unificación
}

\author{
Malte Ebach ${ }^{1}$
}

El llamado a la unificación en biogeografía parece reflejar la frustración de una obvia dicotomía entre los datos y los métodos (Riddle, 2005; Morrone, 2009). Aquellos que enfatizan este problema generalmente tienen poco que decir acerca de cómo se puede promover la unificación tomando en cuenta las discrepancias, y en cambio, intentan crear nuevas síntesis que excluyen una amplia proporción de métodos y por lo tanto crean más divisiones (Donoghue \& Moore, 2003; Crisci et al., 2006; Morrone, 2009). Esto se da porque las síntesis en biogeografía (panbiogeografía, filogeografía), tratan de fundir a los investigadores en torno a un solo método o teoría, en vez de intentar unificar a los biogeógrafos a través de la identificación de problemas biogeográficos, un lenguaje común, y una clasificación universal. Es más, como biogeógrafos, siempre asumimos que nuestro objetivo es el que se tiene que unificar hacia una sola meta.

Problema 1: ¿qué necesita de unificación?

Son los biogeógrafos no la biogeografía quienes necesitan unificarse. Son los biogeógrafos quienes varían en sus objetivos (modelando patrones de distribución, o descubriendo relaciones de área) y sus metas de largo plazo (entendiendo la historia de la distribución de las angiospermas, o definiendo una clasificación biogeográfica de todas las áreas endémicas del Neotrópico). Un filogéografo, por ejemplo, seguramente querrá definir áreas de endemismo y descubrir las relaciones de

\footnotetext{
School of Biological, Earth \& Environmental Sciences, University of New South Wales (Australia). E-mail: mcebach@gmail.com
}

áreas de dos o más genealogías. Incluso un solo biogéografo puede tener dos visiones y objetivos diferentes y conflictivos.

Ponemos mucho énfasis en la metodología. Ello ha llevado a que los métodos definan los objetivos, por ejemplo BPA pretende identificar eventos de dispersión y vicarianza (Wiley, 1987 y todas las posteriores versiones), pero al mismo tiempo es incapaz de reconocer relaciones básicas de áreas (Ebach et al., 2003) ¿Qué pasaría si decidimos descubrir relaciones de áreas o solamente queremos saber qué taxones son partes de eventos amplios de vicarianza? La búsqueda de lo mejor solo funciona si tenemos objetivos y metas concretas en mente. Muchos biogeógrafos no tienen objetivos sólidos o metas de largo plazo, enfocándose más bien en la información extra que puede ser obtenida de un solo taxón, ya sea tratándose de datos filogenéticos o de distribución o ambos. Pocos biogeógrafos hacen biogeografía para abordar problemas biogeográficos generales.

\section{Problema 2: ¿cuáles son los "grandes" problemas biogeográficos?}

Los problemas principales son parte de estudios grandes; aparecen frecuentemente en la literatura trabajos que intentan responder cuestiones importantes con datos limitados (por ejemplo, con un solo taxón):

- Un análisis filogenético a lo largo de tres provincias biogeográficas en el Pacífico Suroriental: el caso del gastrópodo marino Concholepas Concholepas (Cárdenas et al., 2009).

- El archipiélago hawaiano actúa como stepping stone de dispersión en el Pacífico: 
un ejemplo del género de plantas Melicope (Rutaceae) (Harbaugh et al., 2008).

- El origen y evolución de la biotas de las islas indomalayas australasianas y pacíficas: visión desde Aglaieae (Meliaceae, Sapindales) (Muellner et al., 2008).

En otros casos, las preguntas son muy generales o vagas (una síntesis basada en un simple mecanismo o supuesto):

- ¿Llevado por el viento del oeste? Una síntesis de la biogeografía marina surtemplada, con nuevas direcciones para el dispersalismo (Waters, 2008).

- La deriva del viento del oeste revisitada: probando la dispersión dirigida en el hemisferio Sur usando parsimonia de procesos (event-based tree fitting) (Sanmartín et al., 2007).

Llegar a identificar áreas endémicas en el Pacífico, por ejemplo, es un desafío enorme que requiere muchos más datos que por ejemplo una sola filogenia, no importando cuán amplio sea el taxón.

Si bien los artículos destacados arriba pretenden resolver grandes preguntas, aparentan integrar más conocimiento del que los autores realmente poseen. Por ejemplo, en el artículo que aborda la deriva del viento del oeste, los autores no se preocupan de definir las áreas minuciosamente (ellos usan Australia desde varias fuentes pero nunca la definen); tampoco investigan los remolinos oceánicos en la Corriente Circumpolar Antártica (que corren de este a oeste en el mar de Tasmania; Rintoul et al., 2001). Grandes problemas requieren grandes análisis. Resolver los patrones del hemisferio Sur sobre la base de un solo método (en este caso el programa DIVA, que es pobre en detectar relaciones de áreas) y datos arbitrarios (áreas débilmente definidas) solo debilita el argumento. Los biogeógrafos no pueden resolver problemas grandes individualmente o dentro de grupos de taxones específicos. Otro caso, podemos tomar cuatro géneros de trilobites para intentar resolver la paleogeografía del Devónico medio (Ebach \& Edgecombe, 2001; Humphries \& Ebach, 2004); a pesar de que la intención de los autores era el mostrar que las filogenias de trilobites pueden ser usadas para mostrar relaciones de áreas del pasado y responder algunos problemas biogeográficos, hubiese sido ideal el uso de filogenias de peces placodermos, braquiópodos, conodontos y corales.

Es obvio que hay muchas preguntas grandes en biogeografía, así como las hay en filogenética, sin embargo, pocos sistemáticos usarían un simple carácter, por ser dentición, para realizar hipótesis acerca de las relaciones sistemáticas dentro de una clase animal como los mamíferos. Pero esto es exactamente lo que los biogeógrafos intentan hacer.

\section{Problema 3: ¿qué podemos hacer para entendernos?}

La comunicación en cualquier campo científico es indispensable para establecer metas comunes y esbozar divisiones distintivas. Estas metas y distinciones se hacen aparentes en el nivel de la clasificación y nomenclatura. Por ejemplo el nombre 'Australia' no identifica un área simple. En efecto, el nombre está ligado a numerosas definiciones de áreas que se superponen a los límites geopolíticos del país (Williams \& Ebach, 2007; Ebach et al., 2008).

La imposibilidad de distinguir una definición de la otra lleva a la ruptura de la comunicación. En análisis biogeográficos ello significa que necesitamos redefinir todo cada vez, obteniendo siempre resultados diferentes (Ebach et al., 2008). Ligando un nombre a una definición de la misma forma en que un nombre de un taxón a una diagnosis crea un sistema de nominación coherente o nomenclatura. Tomemos por ejemplo la palabra bestia. Puede referirse a cualquier animal grande, lagarto, camión o motocicleta. La palabra es vaga y no puede ser usada en una filogenia. ¿Con que estará relacionada la bestia? Si usáramos el nombre para designar a un solo taxón como bestia, entonces sabríamos a qué nos referimos. Australia se refiere a un número de áreas diferentes. Sin una nomenclatura, como una diagnosis, los biogeógrafos no podrán comunicarse y las clasificaciones se harán imposibles. 


\section{Problema 4: ¿cómo construir una} clasificación biogeográfica universal?

La historia de la biogeografía tiene sus raíces en esquemas de clasificación de plantas y animales (Ebach \& Goujet, 2006). La combinación de estos esquemas por Wallace (1876) ha generado una profusión de esquemas de clasificación revisados para animales, plantas y una combinación de ambos. De hecho, no hay una única clasificación sino muchas, que son todas igualmente válidas. Los taxónomos han reconocido los pros y contras de numerosos esquemas de clasificación para plantas, animales, plantas cultivadas y bacterias. La búsqueda de clasificaciones naturales y el uso de datos moleculares han creado más variaciones, las cuales han sido aceptadas bajo los actuales códigos de nomenclatura y son por lo tanto válidas. Los no taxónomos que quieren usar una clasificación única encuentran dichas taxonomías inútiles y frustrantes. Actualmente tenemos problemas, pero el caso de la taxonomía, hay una necesidad de varias clasificaciones con la meta de largo plazo de una clasificación natural común. Dicha meta de largo plazo no es una taxonomía unitaria dogmática (Godfray, 2007), sino una que puede acomodarse para hipótesis conflictivas.

El concepto de clasificación de áreas bióticas en vez de regiones geográficas usa distribuciones táxicas comunes y las barreras antiguas y actuales que definen distribuciones pasadas y futuras (Parenti \& Ebach, 2009). Esto significa que las áreas bióticas se superponen en el tiempo y el espacio creando coberturas de mapas en vez de provincias biogeográficas estrictamente definidas, como las de Udvardy (1975), o las ecorregiones terrestre marinas o acuáticas del mundo (Olson et al., 2001; Spalding et al., 2007; Abell et al., 2008). Es más, una clasificación de áreas bióticas consistiría en diferentes mapas que se correlacionan con las reconstrucciones paleogeográficas.

\section{Hacia la unificación de los biogeógrafos}

Los mencionados problemas podrían ser resueltos en cuatro formas:

\section{Solución 1: formar grandes grupos de trabajo en biogeografía}

Los geólogos son conocidos por formar grandes grupos de trabajo, como en el marco de los Congresos Internacionales de Geología. La premisa tras estos grandes grupos de trabajo es que se debe abordar un problema particular a la vez. Por ejemplo el grupo IGCP 503 de Paleogeografía y Paleoclima del Ordovícico está compuesto por 268 miembros de 37 países $^{2}$. Ningún miembro está empleado a tiempo completo, sin embargo, dona su tiempo al proyecto. Los biólogos están tomando parte en proyectos similares, como el proyecto El Árbol de la Vida (A Tree of Life: ATol). Los biogeógrafos también pueden organizarse en grupos que persigan una meta común, como podría ser la biogeografía del sudeste asiático, implementando diferentes objetivos, como la clasificación de diferentes áreas bióticas o los trazos de distribución de peces marinos. Los grandes grupos de trabajo son esenciales para la sobrevivencia de la biogeografía como un campo independiente capaz de formular grandes preguntas.

\section{Solución 2: identificar problemas biogeográficos}

Hay un número de problemas biogeográficos en biogeografía que benefician otros campos. En el pasado, por ejemplo, la biogeografía ha aportado la única evidencia concreta para la teoría de la deriva continental. Actualmente muchos biogeógrafos sienten que la biogeografía tiene poco que ofrecer al aporte de evidencias. Un estudio de Escalante et al. (2007), sin embargo, ha aportado evidencia que sugiere que la Placa Caribeña habría evolucionado en forma separada a la coalición entre los márgenes continentales de América del Norte y del Sur durante el Paleoceno (65 a $60 \mathrm{Ma}$ ). Dicha

2 Para Mayor información: http://sarv.gi.ee/igcp503/ 
evidencia es un aporte al largo debate acerca de si la placa del Caribe acrecionó in situ o derivó desde el Pacífico. La evidencia biogeográfica sustenta el mencionado 'Modelo Pacífico', pues la biota caribeña es distinta de la biota neotropical o neártica, las cuales están más estrechamente relacionadas entre sí. Esto puede solo suceder si la placa derivó desde otro lugar, mientras que una placa acrecionada in situ mostraría mayores similitudes entre las biotas caribeña y las antes mencionadas. Por otro lado, resolver la biogeografía del sudeste asiático ayudaría a resolver largas controversias acerca de la evolución geológica del Indo-Pacífico (Parenti \& Ebach, 2009). Lo mismo es válido para el sureste de Australasia.

De hecho, la biogeografía tiene mucho más que ofrecer en escalas amplias a disciplinas como la geología, paleogeografía y paleoclimatología. Es más, muchas reconstrucciones paleomagnéticas dependen fuertemente de datos ad hoc para estimar paleolongitudes. Grandes grupos de trabajo en biogeografía que pregunten grandes interrogantes serán capaces de aportar respuestas o al menos comparar las evidencias entre teorías en conflicto.

\section{Solución 3: usar una clasificación biogeográfica universal}

Sin un sistema de clasificación, la filogenética sucumbiría como ciencia. Lo mismo sucede con la biogeografía. Si no conocemos las áreas que estudiamos, nuestros resultados no tendrán ningún significado. En forma independiente, muchos biogeógrafos han compilado diversos sistemas de clasificación (Ebach \& Goujet, 2006); estos son de naturaleza variable: algunos son jerárquicos como el de Sclater (1858), otros carecen de jerarquía como el de Merrian (1892). En cualquier caso, cada área es definida como una región en el mapa o por sus coordenadas geográficas. Nuestras hipótesis biogeográficas se relacionan con estas áreas y con los taxones que en ellas viven, de forma que una región geopolítica única como Argentina, puede tener una clasificación para peces continentales (López et al., 2008) y otra muy diferente para plantas (Roiga et al., 2009). Un sistema de clasificación preliminar que incorpore estos dos muy diferentes conceptos taxonómicos sería ideal para acomodar visiones diferentes o incluso opuestas, tal como hacen las taxonomías botánicas y zoológicas existentes. Estas clasificaciones pueden ser documentadas y chequeadas para ver qué regiones y áreas son parte de una clasificación natural. Este proceso aplicable en dos pasos partiría por documentar el set actual de clasificaciones diversas. El segundo paso es chequear estas clasificaciones y obtener las áreas bióticas naturales para establecer una jerarquía que conforme un sistema común de clasificación biogeográfica. Dicha clasificación proveerá un sistema de áreas y regiones más estable y riguroso que el actual sistema de ecorregiones del mundo. También será un recurso útil para los no biogeógrafos que requieren dichos datos (mapas) para identificar hotspots de endemismo a mayor detalle o calcular estimaciones de biodiversidad. Más importante, los nombres de dichas áreas deben ser definidos sobre la base de una nomenclatura estable para evitar ambigüedades y duplicaciones.

\section{Solución 4: usar el código internacional de nomenclaturas de áreas (ICAN)}

El código internacional de nomenclaturas de áreas (ICAN) ha sido exitosamente usado en biogeografía desde su establecimiento desde 2007 (Ebach et al., 2008; Parenti et al., 2009; López et al., 2008; Parenti \& Ebach, in press). EI ICAN es un simple y efectivo sistema de nomenclatura que puede no ser familiar para personas ajenas a la sistemática y taxonomía. Resuelve el problema de nombres duplicados para la misma área o el mismo nombre para áreas diferentes. También resuelve qué nombres tienen la prioridad, lo que significa que cada vez que se propone un área nueva, necesita ir acompañada de una diagnosis. Las áreas propuestas tendrán que ser citadas de esta forma, de manera que quien lea el estudio sepa exactamente a cuál Australia se refiere el autor. El ICAN, usado en una clasificación biogeográfica, ayudará a los biogeógrafos a construir una taxonomía más estable no afecta a las ambigüedades nomenclaturales. Más importante, ayudará a los biogeógrafos a comunicarse sin necesidad de reiteración. 


\section{Consideraciones finales}

La biogeografía es una relevante disciplina científica que hace importantes preguntas. Estas han ayudado a resolver otras interrogantes de la biología y de las ciencias de la Tierra. En el estado actual, la biogeografía se encuentra debilitada por la inhabilidad de los biogeógrafos para organizarse en grandes grupos de trabajo con objetivos concretos. Es más, los biogeógrafos usan clasificaciones de áreas pero rara vez buscan encontrar un sistema de clasificación biogeográfica común que pueda ser usado por todos. Actualmente las ciencias biológicas y de la Tierra necesitan avanzar en las grandes respuestas y un sistema de clasificación biogeográfico estable para progresar. Como biogeógrafos podemos abordar estas demandas si nos organizamos, formulamos grandes preguntas y construimos un sistema común de clasificación biogeográfica. ¡Biogeógrafos del mundo, uníos!

\section{Referencias bibliográficas}

ABELL, R.; THIEME, M.; REVENGA, C.; BRYER, M.; KOTTELAT, M.; BOGUTSKAYA, N.; COAD, B.; MANDRAK, N.; CONTRERASBALDERAS, S.; BUSSING, W.; STIASSNY, M. L. J.; SKELTON, P.; ALLEN, G. R.; UNMACK, P.; NASEKA, A.; NG, R.; SINDORF, N.; ROBERTSON, J.; ARMIJO, E.; HIGGINS, J.; HEIBEL, T. J.; WIKRAMANAYAKE, E.; OLSON, D.; LOPEZ, H. L.; REIS, R. E. D.; LUNDBERG, J. G.; SABAJ PEREZ, M. H. \& PETRY, P. Freshwater Ecoregions of the world: A new map of biogeographic units for freshwater biodiversity conservation. BioScience, 2008, No 58, p. 403-414.

CÁRDENAS, L.; CASTILLA, J. C. \& VIARD, F. A phylogeographic analysis across three biogeographic provinces of the Southeastern Pacific: the case of the marine gastropod Concholepas concholepas. Journal of Biogeography, 2009, No36, p. 969-981.

CRISCI, J. V.; SALA, O. E.; KATINAS, L. \& POSADAS, P. L. A. S. Johnson Review $N^{\circ} 4$. Bridging historical and ecological approaches in biogeography. Australian Systematic Botany, 2006, № 19, p. 1-10.
DONOGHUE, M. J. \& MOORE, B. R. Toward an integrative historical biogeography. Integrative and Comparative Biology, 2003, No 43, p. 261-270.

EBACH, M. C. \& EDGECOMBE, G. D. Cladistic biogeography: component-based methods and paleontological application. In: ADRAIN, J. M.; EDGECOMBE, G. D. \& LIEBERMAN, B. S. (eds.). Fossils, phylogeny and form: an analytical approach. New York: Plenum, 2001, p. 235-289.

EBACH, M. C. \& GOUJET, D. The first Biogeographical Map. Journal of Biogeography, 2006, No33, p. 761-769.

EBACH, M. C; HUMPHRIES C. J. \& WILLIAMS, D. M. Phylogenetic biogeography deconstructed. Journal of Biogeography, 2003, No 30, p. 1285-1296.

EBACH, M. C.; MORRONE, J. J.; PARENTI, L. R. \& VILORIA Á. L. International Code of Area Nomenclature. Journal of Biogeography, 2008, No 35, p. 1153-1157.

ESCALANTE, T.; RODRÍGUEZ, G.; CAO, N.; EBACH, M. C. \& MORRONE, J. J. Cladistic biogeographic analysis suggests a Caribbean diversification prior to the Great American Biotic Interchange and the Mexican Transition Zone. Naturwissenschaften, 2007, No 94, p. 561-565.

GODFRAY, H. C. J. Linnaeus in the information age. Nature, 2007, $N^{\circ}$ 446, p. 259-60.

HARBAUGH, D. T.; WAGNER, W. L.; ALLAN, G. J. \& ZIMMER, E. A. The Hawaiian archipelago is a stepping stone for dispersal in the Pacific: an example from the plant genus Melicope (Rutaceae). Journal of Biogeography, 2008, No 36, p. 230-241.

HUMPHRIES, C. J. \& EBACH, M. C. Biogeography on a Dynamic Earth. In: M. LOMOLINO \& BROWN, J. (eds.). The foundations of biogeography. Massachusetts: Sinauer Press, 2004, p. 67-86.

LÓPEZ, H. L.; MENNI, R. C.; DONATO, M. \& MIQUELARENA, A. M. Biogeographical 
revision of Argentina (Andean and Neotropical Regions): an analysis using freshwater fishes. Journal of Biogeography, 2008, vol. 35, № 9, p. 1564-1579.

MERRIAM, C. H. The geographical distribution of life in North America with special reference to the Mammalia. Proceedings of the Biological Society of Washington, 1892, $\mathrm{N}^{\circ} 7$, p. 1-64.

MORRONE, J. J. Evolutionary biogeography: an integrative approach with case studies. Chicago: The University of Chicago Press, 2009.

MUELLNER, A. N.; PANNELL, C. M.; COLEMAN, A. \& CHASE, M. W. The origin and evolution of Indomalesian, Australasian and Pacific island biotas: insights from Aglaieae (Meliaceae, Sapindales). Journal of Biogeography, 2008, № 35, p. 1769-1789.

OLSON, D. M.; DINERSTEIN, E.; WIKRAMANAYAKE, E. D.; BURGESS, N. D.; POWELL, G. V.; UNDERWOOD, E. C.; D'AMICO, J. A.; ITOUA, I.; STRAND, H. E.; MORRISON, J. C.; LOUCKS, C. J.; ALLNUTT, T. F.; RICKETTS, T. H.; KURA, Y.; LAMOREUX, J. F.; WETTEN-GEL, W. W.; HEDAO, P. \& KASSEN, K. R. Terrestrial ecoregions of the world: A new map of life on Earth. Bioscience, 2001, № 51, p. 933-938.

PARENTI, L. R.; VILORIA, Á. L.; EBACH, M. C. \& MORRONE, J. J. On the International Code of Area Nomenclature (ICAN): a Reply. Journal of Biogeography, 2009, $\mathrm{N}^{\circ} 36, \mathrm{p}$. 1619-1621.

PARENTI, L. R. \& EBACH, M. C. Comparative Biogeography: Discovering and classifying biogeographical patterns of a dynamic Earth. Berkeley: University of California Press, 2009.

PARENTI, L. R. \& EBACH, M. C. Wallacea Deconstructed. In: WILLIAMS, D. M. \& KNAPP, S. (eds.). Beyond Cladistics. Berkeley: University of California Press, in press.

RIDDLE, B. R. Is biogeography emerging from its identity crisis? Journal of Biogeography, 2005, № 32, p. 185-186.
RINTOUL, S.; HUGHES, C. \& OLBERS, D. The Antarctic Circumpolar Current System. Ocean Circulation and Climate. International Geophysics Series, 2001, vol. 77, p. 271-302.

ROIGA, F. A.; ROIG-JUÑENTB, S. \& CORBALÁNB, V. Biogeography of the Monte Desert. Journal of Arid Environments, 2009, $N^{\circ} 73$, p. 164-172.

SANMARTÍN, I.; WANNTORP, L. \& WINKWORTH, R. C. West Wind Drift revisited: testing for directional dispersal in the Southern Hemisphere using event-based tree fitting. Journal of Biogeography, 2007, $\mathrm{N}^{\circ}$ 34, p. 398-416.

SCLATER, P. L. On the general geographical distribution of the members of the class Aves. Proceedings of the Linnean Society (Zoology), 1858, № 2, p. 130-145.

SPALDING, M. D.; FOX, H. E.; ALLEN, G. R.; DAVIDSON, N.; FERDAÑA, Z. A.; FINLAYSON, M.; HALPERN, B. S.; JORGE, M. A.; LOMBANA, A.; LOURIE, S. A.; MARTIN, K. D.; MCMANUS, E.; MOLNAR, J.; RECCHIA, C. A. \& ROBERTSON, J. Marine ecoregions of the world: a bioregionalization of coastal and shelf areas. Bioscience, 2007, $N^{\circ} 57$, p. 573-583.

UDVARDY, M. D. F. A classification of the biogeographical provinces of the world. Morges: IUCN Occasional Papers, International Union for Conservation of Nature and Natural Resources, 1975.

WALLACE, A. R. The geographical distribution of animals; with a study of the relations of living and extinct faunas as elucidating the past changes of the Earth's surface. London: Macmillan, 1876.

WATERS, J. M. Driven by the West Wind Drift? A synthesis of southern temperate marine biogeography, with new directions for dispersalism. Journal of Biogeography, 2008, $\mathrm{N}^{\circ} 35$, p. $417-427$

WILLIAMS, D. M. \& EBACH, M. C. Foundations of Systematics and Biogeography. New York: Springer, 2007. 